\title{
Parallelized Hybrid TGRAPPA Reconstruction for Real-Time Interactive MRI
}

\author{
Haris Saybasili ${ }^{1,2}$, Peter Kellman ${ }^{1}$, J. Andrew Derbyshire ${ }^{1}$, Elliot R. McVeigh ${ }^{3}$, \\ and Michael A. Guttman ${ }^{1}$ \\ ${ }^{1}$ NHLBI, National Institutes of Health, DHHS, Bethesda, MD, USA \\ saybasilih@mail.nih.gov \\ ${ }^{2}$ Bogazici University, Biomedical Engineering Institute, Istanbul, Turkey \\ 3 Johns Hopkins University, Dept. of Biomedical Engineering, Baltimore, MD, USA
}

\begin{abstract}
Real-time parallel MRI reconstruction was demonstrated using a hybrid implementation of the TGRAPPA algorithm. The GRAPPA coefficients were calculated in $\mathrm{k}$-space and applied in the image domain after appropriate transformation, thereby achieving improved speed and excellent image quality. Adaptive B1-weighted combining of the per coil images permitted use of pre-calculated composite image domain weights providing significant decrease in computation. The weight calculation was decoupled from the real-time image reconstruction as a parallel processing thread which was updated in an adaptive manner to speed convergence in the event of interactive change in scan plane. The computation was parallelized and implemented on a general purpose multicore architecture. Reconstruction speeds of 65-70 frames per second were achieved with a matrix of $192 \times 144$ with 15 coils.
\end{abstract}

\section{Introduction}

Parallel MRI (pMRI) methods aim to increase the imaging speed without degrading temporal resolution, by the use of multiple receiver coils and partial $\mathrm{k}$-space coverage. Each of these coils is placed at different locations on the patient, thus sensing the MR signal with different spatial sensitivities. Therefore, each MRI receiver acquires data corresponding to an aliased image, modulated by its unique spatial sensitivity pattern. Knowledge of the sensitivity information can be used to correct the aliasing, or equivalently, to synthesize data for the skipped spatial encoding steps. Several pMRI methods have been proposed including the well-known image domain SENSE [1] and k-space domain GRAPPA 2] algorithms.

The proposed approach uses the GRAPPA algorithm for k-space weight calculation since this method is tolerant of prefolding (or wrap) within the user defined FOV, which may be advantageous when small FOV is desired. Autocalibration was performed using a time interleaved acquisition scheme as done in TSENSE 3 by combining $\mathrm{R}$ (acceleration factor) consecutive frames to form a low temporal resolution full k-space dataset for weight calculation as done in TGRAPPA [4]. 
Although TSENSE has been deployed in real time interventional applications for several years [5], to the best of our knowledge TGRAPPA has not yet been demonstrated with real-time, low latency image reconstruction. The TGRAPPA algorithm uses convolution-like operations to estimate the skipped k-space data, requiring significantly greater computation than TSENSE. The calculation of weights as well as the application of weights are both performed in k-space. However, the convolution operation in the $\mathrm{k}$-space domain is equivalent to simple multiplication in the image-domain. Thus, it is possible to perform a hybrid k-space and image-domain reconstruction [6, which will greatly reduce computation and remove dependency of reconstruction speed on kernel size and acceleration rate.

(T)GRAPPA reconstruction is performed on a per coil basis and combined using root sum of squares in order to avoid dephasing. In this work, we use adaptive B1-weighted combining [7] primarily for computational speed. In this approach, a composite set of unmixing coefficients are calculated that linearly combines the per coil coefficients with an estimated sensitivity map. These pre-calculated weights are applied to the aliased images to reconstruct a single unaliased image (as in SENSE) resulting in a significant computational reduction.

In this work, we demonstrate a parallelized hybrid TGRAPPA implementation with simplified computation and continuously updated auto-calibration for real time interactive MRI.

\section{Method}

In (T)GRAPPA, the missing data for a particular coil is estimated from all the acquired data from all the coils. Suppose $N_{c}$ is the number of receiver coils and $R$ is the acceleration rate. For each acquired k-space point from each coil $\left(c, k_{x}, k_{y}\right)$ it is necessary to synthesize $(R-1)$ sample estimates to completely fill out the acquisition matrix. Blocks of $[Y \times X]$ neighboring acquired samples from all the receiver coils are used to obtain estimates of these missing samples for a given coil. The choice of block size affects the compromise between image quality and reconstruction speed. Figure 1 shows examples of $[4 \times 5]$ and $[2 \times 5]$ blocks at one k-space location with the missing $\mathrm{k}$-space samples for $R=3$.

Consider a particular block for a particular coil $c_{k}$. The $R-1$ unknown points in the block are estimated as a linear combination of the known samples in the corresponding blocks from all the receiver coils. For this, each missing sample in the block requires $\left(X Y N_{c}\right)$ weighting coefficients. Let $S(c, i, j)$ be the $(i, j)$ element of the acquired data in the corresponding block of data from coil c (i.e. the square samples in Fig 1$)$. Let $W_{c_{k}, p}(c, i, j)$ be the set of GRAPPA weight coefficients used to weight the samples $S(c, i, j)$ in order to synthesize $T_{c_{k}, p}$, the $p$ th missing sample of the target block $c_{k}$ (i.e. the stars in Fig 1). This relation can be expressed as

$$
\sum_{c=1}^{N_{c}} \sum_{i=1}^{X} \sum_{j=1}^{Y} S(c, i, j) W_{c_{k}, p}(c, i, j)=T_{c_{k}, p}
$$




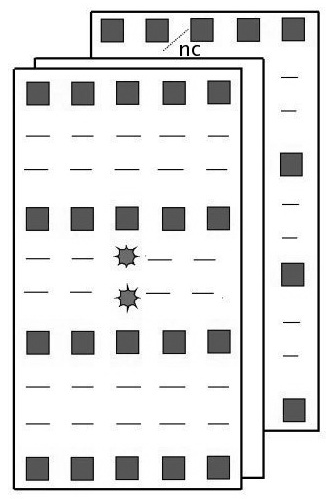

a.
:Known points

: Missing points nc: Number of coils

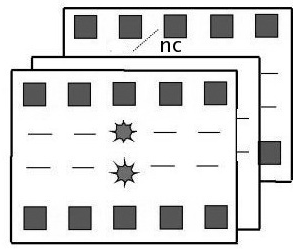

b.

Fig. 1. a. $R=3$, block of size $4 \times 5$ (4 lines, 5 points per line). b. $R=3$, block of size $2 \times 5$ (2 lines, 5 points per line).

The weighting coefficients $W_{c_{k}, p}(c, i, j)$ are obtained from the fully sampled auto-calibration data by solving the equation Eq. 1 using least squares estimation of the overdetermined system formed by considering all the blocks in the central parts of k-space. The precision of the weight-sets increases by increasing the number of blocks to use in the estimation.

Calculation of the weight-sets is computationally demanding, but needs only to be performed infrequently. The resulting weights may be re-used for multiple frames of reconstruction until the image encoding process changes (e.g. due to a change of scan plane or movement of the receiver coil array).

While estimation of the missing k-space data from acquired data using Eq.11is less demanding than the calculation of the weight-sets, it must be performed in real-time for each image to be reconstructed, and hence determines the effective reconstruction speed. Furthermore, this calculation is significantly more computationally intensive than corresponding calculations for image-based parallel imaging methods (e.g. (T)SENSE) because it employs a convolution type process at each sample, as opposed to a pixel-by-pixel multiplication. Furthermore, operating in the k-space domain requires processing of data from all receiver channels, thereby increasing demands on computation and data management. For TGRAPPA, the speed of reconstruction depends on the size of the acquisition matrix; the number of receiver coils $N_{c}$; the acceleration rate, $R$; and the block size, $[Y \times X]$.

We note that Eq. 1 is a convolution-type operation performed in the frequency domain which can be eliminated in favor of a direct multiplication if the TGRAPPA weight-sets are transformed into the image-domain. Thus, it is possible to reduce the dependency of the reconstruction speed to the size of the acquisition matrix and then number of receiver coils $N_{c}$, dramatically improving the reconstruction speed when high acceleration rates and/or large block sizes are used. 


\section{TGRAPPA Reconstruction in the Image Domain}

For image-domain based TGRAPPA reconstruction, the weight-sets must be transformed into the image-domain. This is achieved by combining the $p=[1$ : $R-1$ ] weight-sets $w_{c_{k}, p}(c, \cdot, \cdot)$ (which relate the acquired data in coil c to the missing data from coil $\left.c_{k}\right)$ to yield $N_{c}$ k-space convolution kernels $W_{c_{k} c}^{s e t}\left(k_{x}, k_{y}\right)$ for each coil, $c_{k}$. The centre point of these kernels must be set to $\delta_{c_{k} c}$, corresponding to preserving the acquired samples for the coil $c_{k}$. The kernel is then zero-filled to the same matrix size as the actual images and inverse Fourier transformed into the image-domain.

$$
w_{c_{k} c}^{i m g}=\mathscr{F}^{-1}\left(W_{c_{k} c}^{s e t}\right)
$$

The composite unmixing coefficient $u_{c}^{c o m p}$ of coil $c$ is obtained by pixel-bypixel multiplying each $w_{c_{k} c}^{i m g}$ by complex conjugate of B1-map estimates $b 1_{c_{k}}$, calculated from the temporal average of data from each coil $c$ and summing, thus:

$$
u_{c}^{c o m p}=\sum_{c_{k}=1}^{N_{c}} w_{c_{k} c}^{i m g} \cdot b 1_{c_{k}}^{*}
$$

where $\cdot$ denotes pixel-by-pixel multiplication operator.

For the reconstruction, the under-sampled k-space data, $K_{c}\left(k_{x}, k_{y}\right)$ from each coil, $c=\left[1: N_{c}\right]$, is transformed into image-domain, yielding aliased images $I_{c}^{\text {aliased }}$ :

$$
I_{c}^{\text {aliased }}=\mathscr{F}^{-1}\left(K_{c}\right)
$$

Final image is obtained by

$$
I^{\text {final }}=\sum_{c=1}^{N_{c}} I_{c}^{\text {aliased }} \cdot u_{c}^{\text {comp }}
$$

which is a phased array combiner as in SENSE unmixing. The resulting reconstruction speed does not depend on either $R$ or the block size, and because the convolution operation is replaced by a pixel-by-pixel multiplication, computation is reduced. Multi-coil root sum-of-squares magnitude operation is replaced by a simple linear combination.

\section{Implementation}

The image reconstruction algorithms are implemented in $\mathrm{C} / \mathrm{C}++$ for high performance. Weight-set calculation and image reconstruction were computed in parallel using 8 dual-core AMD Opteron 8220 processor $(2.8 \mathrm{GHz})$ on Linux-2.6.16.460.12-smp. Parallelization of the reconstruction was achieved using Pthreads (http://pasc.org), and parallelization in the weight-set calculation was realized by OpenMP (http://openmp.org). The code compiled on GCC (http://gcc.gnu.org) version 4.2.2. The OpenGL (http://opengl.org) library used for displaying reconstructed images. Matrix inversions, used in the least-squares calculation of the weight-set coefficients from the auto-calibration data, was calculated using the ATLAS library (http://math-atlas. 
sourceforge.net). The FFTW library (http://fftw.org) was used for Fast Fourier Transformations.

Weight-set calculation, the most time consuming step of the whole process, is performed asynchronously to the image reconstruction. During this calculation, image reconstruction with the current weight set updates the auto-calibration data using one thread per coil. At initialization, and later when there is a scanplane change, new weight-sets must be calculated as quickly as possible in order to produce useful images as soon as possible. At that point, the calculation is multithreaded (number of threads = number of CPUs) and a smaller block size is used. This permits images to be reconstructed almost immediately, but with reduced quality. The block size is subsequently increased with each new weight-set calculation to improve the image quality, while the number of threads is decreased to devote more CPU resources to image reconstruction. The use of OpenMP facilitates this strategy since it allows thread count to be modified dynamically.

\section{Results}

Several real-time heart images were acquired using a Siemens Magnetom Espree 1.5T (Siemens Medical Solutions, Erlangen, Germany). The sequence was SSFP 8] with $\mathrm{TR}=3.1$, flip angle $=45$ degrees, bandwith $=800 \mathrm{~Hz} /$ pixel with an acquisition matrix of $192 \times 144$. $\mathrm{S}^{5} \mathrm{FP}$ [9] was used for suppression of fat and flow artifacts. 15 receiver coils were used; 6 elements on the chest and 9 under the spine. The proposed parallel imaging technique, hybrid TGRAPPA, was employed with acceleration factors of $R=2, R=3$, and $R=4$.

\section{Performance}

The performance results for the reconstruction and first time weight-set calculation are given in Table 1 and 2 for both conventional and hybrid TGRAPPA implementation for acceleration rates of $R=2,3,4$, using block sizes of $[2 \times 1]$

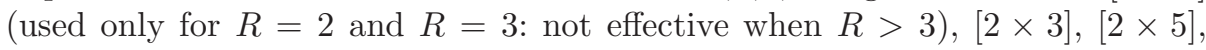
$[2 \times 7],[4 \times 3]$ and $[4 \times 5]$. For both conventional and hybrid TGRAPPA implementations, 60 auto-calibration lines were used for weight-set calculation. Hybrid TGRAPPA weight-set calculation results includes the transformation of the k-space weight-sets into image-domain and the calculation of the composite unmixing coefficients.

\section{Images}

Block sizes of $[2 \times X]$ are preferred in our application as these are computationally less demanding than block sizes of $[4 \times X]$ and provide nearly on par image quality, which can be seen in Fig. 2 that compares rate 4 image with block sizes of $[4 \times 5]$ and $[2 \times 7]$. Figure 3 represents the reconstructed images for $R=3$ for block sizes of $[2 \times 1]$ and $[2 \times 5]$ after hybrid TGRAPPA reconstruction. The smaller sized block gives fast weight-set calculation with reasonable image quality, while bigger sized block gives slow weight-set calculation but superior image quality for a given acceleration rate. Smaller block size causes more artifacts, e.g. see 
Table 1. Image reconstruction times in seconds for conventional (conv.) and hybrid (hyb.) TGRAPPA for different $\mathrm{R}$ values and block sizes of $[2 \times 1],[2 \times 3],[2 \times 5],[2 \times 7]$, $[4 \times 3]$ and $[4 \times 5]$ (acquisition matrix: $192 \times 144, N_{c}=15$ )

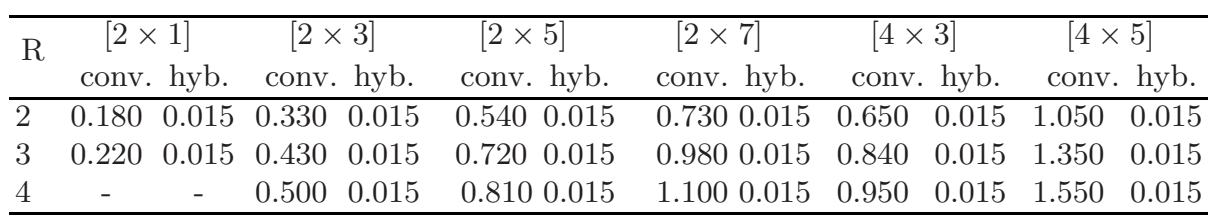

Table 2. First time weight-set calculation times in seconds for conventional (conv.) and hybrid (hyb.) TGRAPPA for different $\mathrm{R}$ values and block sizes of $[2 \times 1],[2 \times 3]$, $[2 \times 5],[2 \times 7],[4 \times 3]$ and $[4 \times 5]$. (acquisition matrix: $192 \times 144, N_{c}=15$ ).

\begin{tabular}{|c|c|c|c|c|c|c|c|c|c|c|c|}
\hline \multirow{2}{*}{$\mathrm{R}$} & \multicolumn{2}{|c|}{$[2 \times 1]$} & \multicolumn{2}{|c|}{$[2 \times 3]$} & {$[2 \times 5]$} & \multicolumn{2}{|c|}{$[2 \times 7]$} & \multicolumn{2}{|c|}{$[4 \times 3]$} & \multicolumn{2}{|c|}{$[4 \times 5]$} \\
\hline & conv. & hyb. & conv. & hyb. & conv. hyb. & conv. & hyb. & conv. & hyb. & conv. & hyb. \\
\hline 2 & 0.080 & 0.400 & 0.270 & 0.580 & 0.6500 .970 & 1.180 & 1.500 & $\overline{0.770}$ & 1.090 & 2.100 & 2.420 \\
\hline & 090 & 10 & 990 & 0 & 01 & 1. & 0 & 20 & 0 & 20 & 2.4 \\
\hline & - & - & 320 & 50 & $\begin{array}{ll}750 & 1.070\end{array}$ & 1.270 & 1.610 & 0.870 & 1.190 & 2.160 & 2.480 \\
\hline
\end{tabular}

the background artifacts and heart muscle wall region marked by white square in Fig. 3. a. Note that in these figures, image brightness levels are increased to make reconstruction artifacts more visible.

\section{Discussion}

Image domain hybrid TGRAPPA reconstruction outperforms conventional kspace TGRAPPA reconstruction as can be seen in Table1 Additionally, imagedomain reconstruction speed remains unaffected by increased acceleration rates and increased block sizes. Thus, the trade-off between reconstruction speed and image quality is eliminated. However, the weight-set calculation slows down due to the additional steps required to transform the k-space weight-sets into the image-domain and to the B1-combining. This slow-down depends on the number of acquisition coils and on image resolution. In our test dataset, the time loss due to the additional transformation was measured as 0.32 seconds, which we considered to be reasonable for our application.

Image domain hybrid TGRAPPA reconstruction image quality is equivalent to conventional TGRAPPA. Additionally, acceleration rate and block size independent reconstruction scheme provide better image quality with faster reconstruction rates. $R=4$ images in Fig. 2 are reconstructed in 0.015 seconds (65-70 frames/sec) which was not previously possible to achieve using conventional TGRAPPA reconstruction on our reconstruction system.

A parallelized hybrid TGRAPPA implementation for real-time applications was developed and demonstrated. For improved reconstruction speeds, k-space domain convolution type operations are instead converted to pixel-wise 

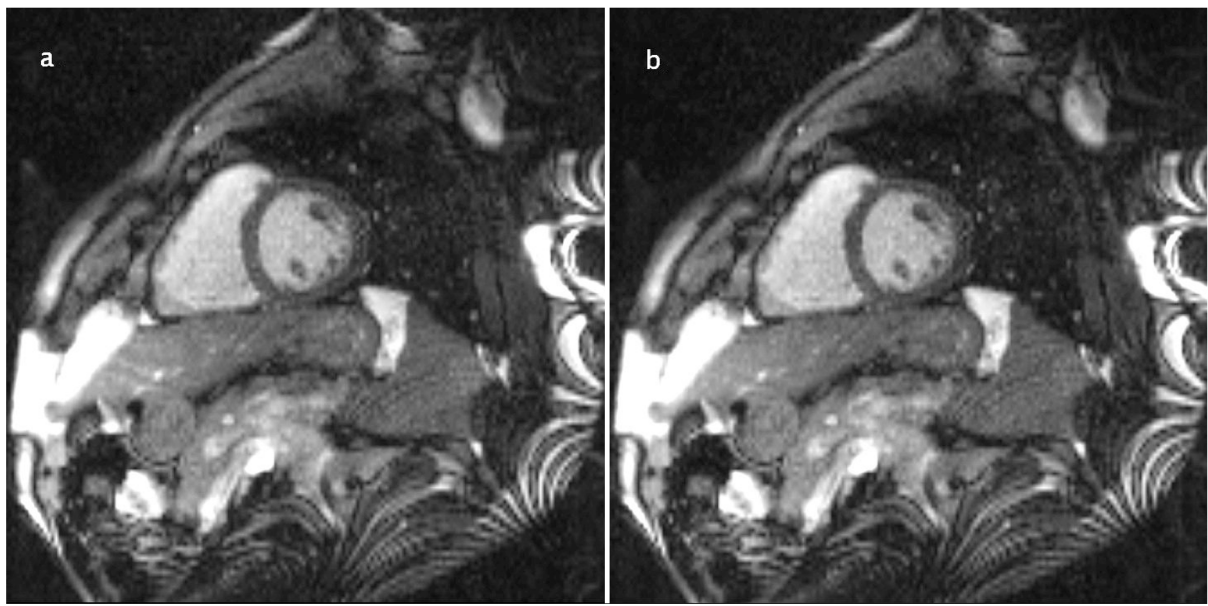

Fig. 2. Image domain hybrid TGRAPPA reconstructed image for $R=4$ (acquisition matrix: $\left.192 \times 144, N_{c}=15\right)$. a. Block size of $[4 \times 5]$ b. Block size of $[2 \times 7]$.

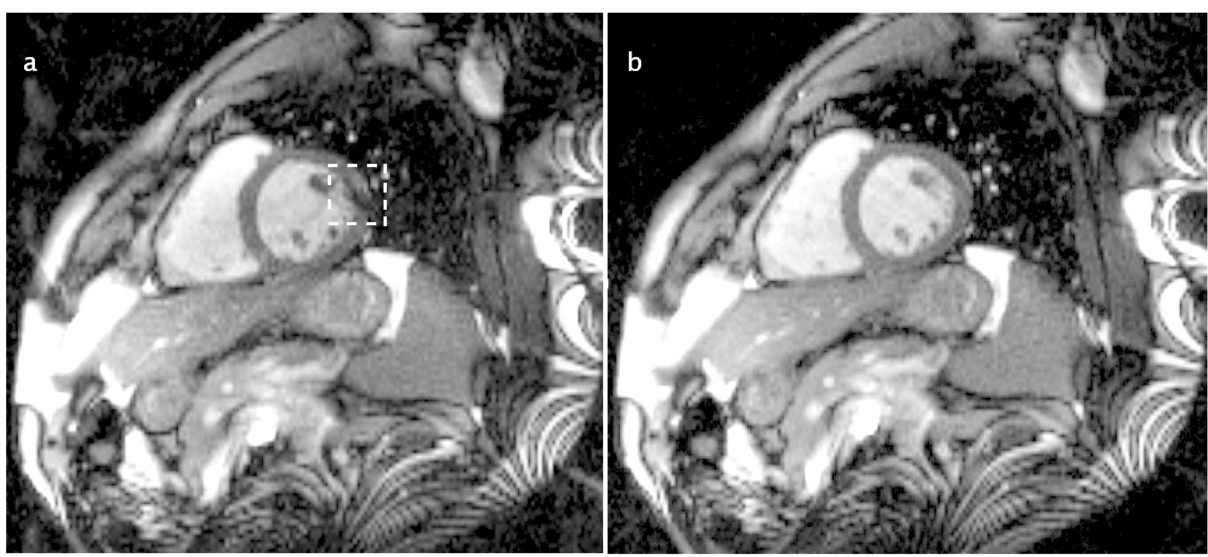

Fig. 3. Image domain hybrid TGRAPPA reconstructed images for $R=3$ (acquisition matrix: $\left.192 \times 144, N_{c}=15\right)$. Image brightness levels are modified for easily viewing the artifacts. a. Block size of $[2 \times 1]$. Artifact in the heart muscle wall is indicated by the dotted white square. Background artifacts can be observed. b. Block size of $[2 \times 5]$.

multiplication in the image domain. This improvement essentially provides a constant reconstruction speed for a given acquisition matrix and number of acquisition coils independent of the acceleration rate and block size. Our approach adds additional steps to the calculation of the weight-sets, however the weight sets are calculated in the background, asynchronous to image reconstruction. Also, by dynamically changing the block size and number of threads to use for weight-set calculation, we can respond to changes in slice plane by first producing 
a lower quality image, then increasing quality for later frames. The weight calculation for the proposed hybrid method was only $20 \%$ slower than conventional (T)GRAPPA, however, the reconstruction speed was up to $100 \mathrm{x}$ faster. This method allows use of the TGRAPPA algorithm for interactive real-time MRI.

\section{References}

1. Pruessmann, K.P., Weiger, M., Scheidegger, M.B., Boesiger, P.: SENSE: Sensitivity Encoding for Fast MRI. Magnetic Resonance in Medicine 42(5), 952-962 (1999)

2. Griswold, M.A., Jakob, P.M., Heidemann, R.M., Nittka, M., Jellus, V., Wang, K., Kiefer, B., Haase, A.: Generalized Autocalibrating Partially Parallel Acquisitions (GRAPPA). Magnetic Resonance in Medicine 47 (2002)

3. Kellman, P., Epstein, F.H., McVeigh, E.R.: Adaptive Sensitivity Encoding Incorporating Temporal Filtering (TSENSE). Magnetic Resonance in Medicine 45(5), 846-852 (2001)

4. Breuer, F.A., Kellman, P., Griswold, M.A., Jakob, P.M.: Dynamic Autocalibrated Parallel Imaging Using Temporal GRAPPA (TGRAPPA). Magnetic Resonance in Medicine 53 (2005)

5. Guttman, M.A., Kellman, P., Dick, A.J., Lederman, R.J., McVeigh, E.R.: Realtime Accelerated Interactive MRI with Adaptive TSENSE and UNFOLD. Magnetic Resonance in Medicine 50(2), 315-321 (2003)

6. Brau, A.C., Beatty, P.J., Skare, S., Bammer, R.: Comparison of Reconstruction Accuracy and Efficiency Among Autocalibrating Data-Driven Parallel Imaging Methods. Magnetic Resonance in Medicine 59, 382-395 (2008)

7. Walsh, D.O., Gmitro, A.F., Marcellin, M.W.: Adaptive Reconstruction of Phased Array MR Imagery. Magnetic Resonance in Medicine 43(5), 682-690 (2000)

8. Oppelt, A., Graumann, R., Barfuss, H., Fischer, H., Hartl, W., Shajor, A.: FISP A New Fast MRI Sequence. Electromedica 54, 15-18 (1986)

9. Derbyshire, J.A., Herzka, D.A., McVeigh, E.R.: S5FP: Spectrally Selective Suppression with Steady State Free Precession. Magnetic Resonance in Medicine 54, 918-928 (2005) 